\title{
A BPM CALIBRATION PROCEDURE USING TBT DATA
}

\author{
M.J. Yang ${ }^{\ddagger}$, J. Crisp, P. Prieto, Fermilab ${ }^{\dagger}$, Batavia, IL 60510, USA
}

\begin{abstract}
Accurate BPM calibration is crucial for lattice analysis. It is also reassuring when the calibration can be independently verified. This paper outlines a procedure that can extract BPM calibration information from TBT orbit data. The procedure is developed as an extension to the TurnBy-Turn lattice analysis [1]. Its application to data from both Recycler Ring and Main Injector (MI) at Fermilab have produced very encouraging results. Some specifics in hardware design will be mentioned to contrast that of analysis results.
\end{abstract}

\section{INTRODUCTION}

The gain of a Beam Position Monitor (BPM) can be calculated from the detector geometry and measured with bench top electro-static style setup [2]. Once installed the gain could be dependent on variations in cabling and on signal processing electronics. Beam based gain calibration is possible when profile monitor with fixed-pitch wire spacing is available. Such is the case only for beam transfer lines. In a circular machine verifying BPM gain is a convoluted exercise and such is the quandary of every machine optics studier. The procedure introduced here provides an independent way to evaluate relative gains of BPMs around the ring with an accuracy of $2 \%$ RMS.

The BPM system upgrade of Fermilab Recycler Ring (RR) was completed by the end of 2004, with single pass resolution around 20 microns in either plane. By design it will only respond to $2.5 \mathrm{MHz}$ beam RF structure. A 2048turn TBT data buffer is built-in for each BPM. The upgrade for Main Injector (MI) BPM system followed and was completed at the end of year 2006. For both machines the upgrades opened up a whole new realm of possibilities and made the analysis presented here possible.

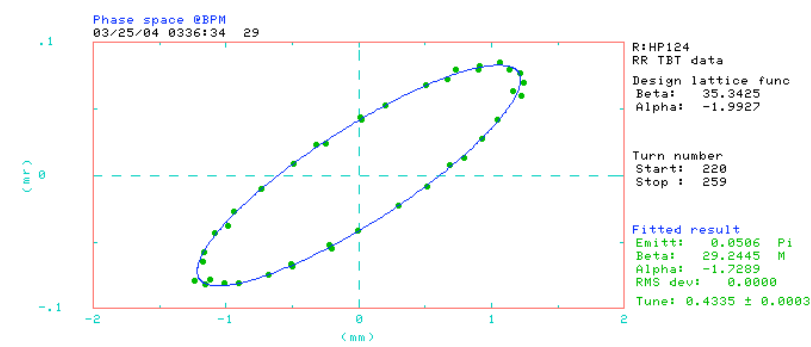

Figure 1. Horizontal plane phase space plot of HP124 in Recycler Ring, for 40 consecutive turns. The measured beta and alpha function is listed at the lower-right corner in green.

\section{ALGORITHM}

The basic concept of this calibration procedure is simple

$\dagger$ Operated by Fermi Research Alliance, LLC under Contract No. DEAC02-07CH11359 with the United States Department of Energy.

¥ E-mail: yang@fnal.gov and can be demonstrated with Figure 1 and 2. In Figure 1 horizontal plane phase-space data points from HP124 are plotted for 40 consecutive turns. The elliptical contour, as shown, is the result of fitting an ellipse to the data points. Parameters from this ellipse lead to the calculation of measured alpha and Beta function, which is also shown at the lower-right corner of the plot. The area encompassed by the ellipse, called emittance $\varepsilon=0.0506 \pi-\mathrm{mm}-\mathrm{mr}$, is also obtained from the fitting algorithm. Being a constant of motion this emittance is used to project oscillation amplitude around the ring according to local beta function at the $i$-th BPM:

$$
\mathcal{A}_{i}=\sqrt{\beta_{i} \times \varepsilon}
$$

One example of actual TBT data from HP222 in Recycler Ring is shown in Figure 2, where measured beta is 46.99 meters. The amplitude and phase of oscillation are extracted through fitting the data points to a sinusoidal waveform, also shown on the plot. Utilizing phase information is a different kind of analysis and is outside the scope of this paper. The fitted amplitude $a_{i}=1.69$ is then compared with that of projected $\mathcal{A}_{i}=1.54$. Nonunity in ratio is attributed to error in gain of the $i$-th BPM and its calibration becomes

$$
g_{i}=\mathcal{A}_{i} / a_{i, \text { measured }} \text {. }
$$

It is important to remember that gain calibration discussed here is only relative, from one BPM to another. Unless the emittance used in the calculation is truly immune to calibration error, oscillation amplitude variation, and others. This procedure is an extension to TBT lattice analysis because of its need for reliable beta functions. In principle, beta functions from any other sources can be used, as long as they are an accurate description of the machine.

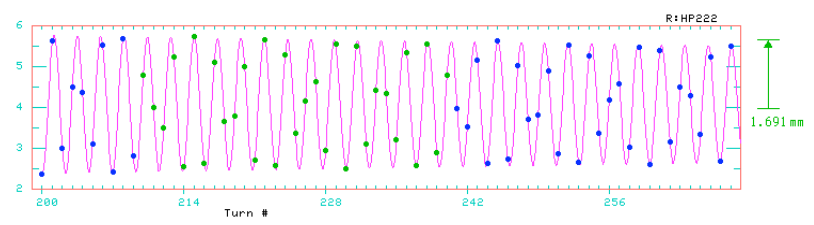

Figure 2. The horizontal plane TBT data at HP222 is plotted for 70 turns. As shown here only the data points in green were used for the sinusoidal fit.

\section{TBT DATA}

With no pinger device in Recycler Ring to excite beam oscillation TBT data had to be taken at injection with intentional steering error in either horizontal or vertical plane. The oscillation amplitudes of TBT data were limited to somewhere between one and two millimeter, for reasons beyond the scope of this paper.

In the Main Injector Ring extraction kicker at MI52 was used to excite horizontal plane oscillation and injection 
kicker at MI10 was used to kick beam in the vertical plane. Typical size of oscillation, in the data taken for study, is around $5 \mathrm{~mm}$.

\section{ANALYSIS RESULTS}

\section{Recycler Ring}

Two different styles of BPM detectors are used in the Recycler Ring. The majority of them are made of diagonally split elliptical beam pipes. Large aperture BPMs, also called $8-\mathrm{GeV}$ style for historical reasons, are made of split round beam pipe. They are used in the two long straight sections, i.e. in RR30 where Electron Cooling for Anti-proton is located and in RR60 where phase trombone is implemented. Because of differences in aperture and signal responses each style of hardware requires its own set of calibration constants.

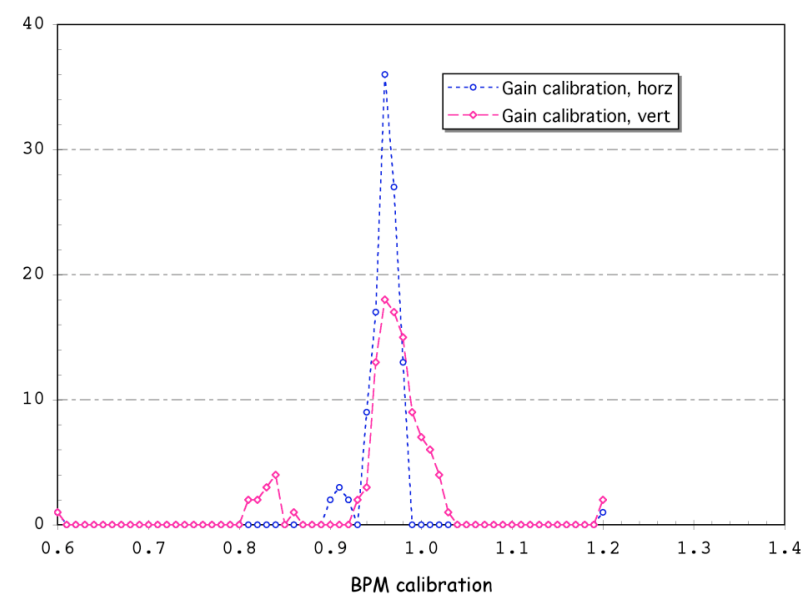

Figure 3. Distribution of Recycler BPM gains, measured soon after the new system was commissioned. Blue is for horizontal plane and magenta is for vertical plane.

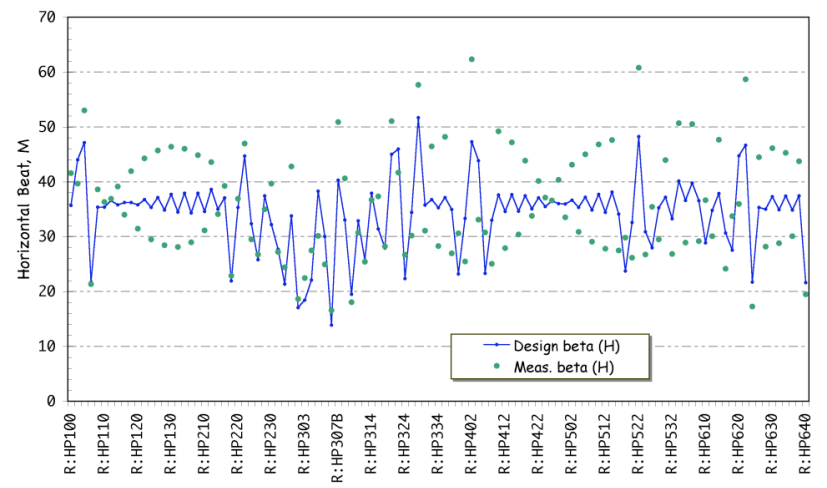

Figure 4. Horizontal plane beta function at the BPMs in Recycler Ring. The design beta functions are plotted in connected blue lines. Green dots are the TBT lattice measurement results that were used for BPM gain calculation.

Soon after the installation of new Recycler BPM system a set of TBT data was taken for lattice analysis, and gain calibration analysis as well. The gain distribution found is shown in Figure 3 with the horizontal plane plotted in blue and the vertical plane in magenta. There are two visi- visible peaks in each plane, one much bigger than the other. A closer look at the result identified that all large aperture BPMs, and only them, are associated with the small peak. To demonstrate how well this procedure worked, the measured horizontal beta functions at all horizontal BPM locations in Recycler Ring are plotted in Figure 4. The measured beta functions are in green dots and the design values in connected blue line. It would be unthinkable if design values had been used instead, when differences in beta between measured and design are as large as $30 \%$.

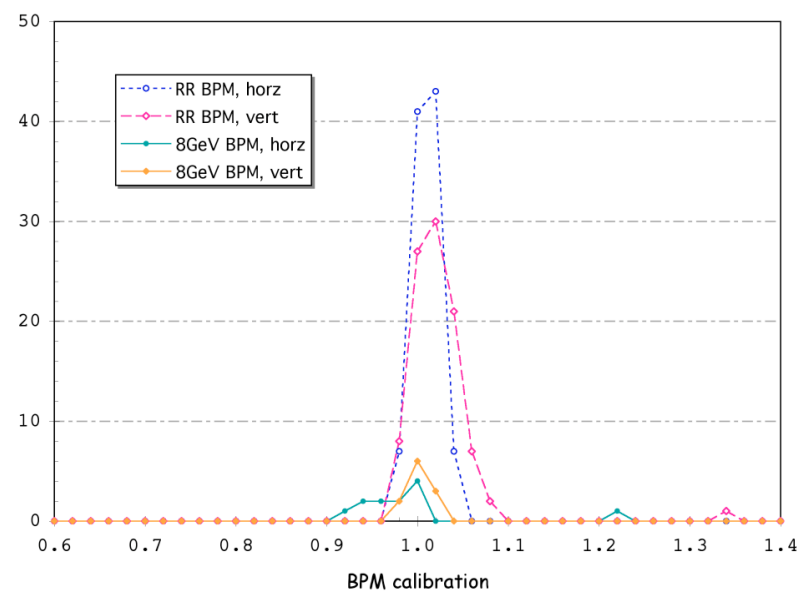

Figure 5. Distribution of Recycler BPM gains calibration measured after gain adjustment to large aperture BPMs, the 8$\mathrm{GeV}$ style, was made. The calibration for these $8 \mathrm{GeV}$ style BPMs are plotted in separate series.

Independently, it was realized that BPM gains had not been set properly during upgrade and were subsequently updated. The analysis result with data taken afterward is shown in Figure 5, in four series. The gains of large aperture BPMs are now in separate series, as they no longer are separable from the larger peak.

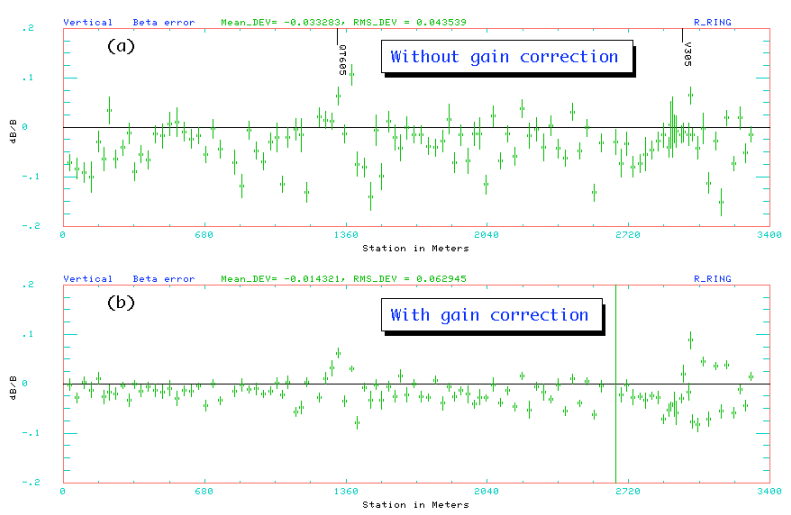

Figure 6. Results of vertical beta function measurement and plotted as $\Delta \beta / \beta$. In plot (a) is the case without applying gain calibration and plot (b) with gain calibration. The measurement is based on 1-bump closed orbit with multiple corrector dipoles, not the TBT analysis as mentioned.

Another success example is shown in Figure 6, where the results of a closed orbit based beta function measurement are plotted. In (a) is the measured vertical beta func- 
tion without any correction to BPM gains and in (b) with gain calibration. This beta measurement utilizes closed orbit responses to single dipole kicks, from two correctors at approximately $90^{\circ}$ apart in phase advance. Its result is known to depend on the square of calibration error and the improvement between the two is substantial.

\section{Main Injector}

Main Injector style BPM is used throughout the ring except at Lambertson locations, where large aperture BPMs are used. The cross-section view of the MI BPM is shown in Figure 7, with four strip-line pickups inside an elliptical beam pipe. The BPM responses, measured with source moving at fixed $5 \mathrm{~mm}$ steps in both plane, are shown in red circles which formed a distorted grid. The distortion is due to non-linear response of the BPM whose gain varies according to beam position in the other dimension. A fact that is ignored by the BPM system but reflected in the BPM gain analysis result and will be discussed.

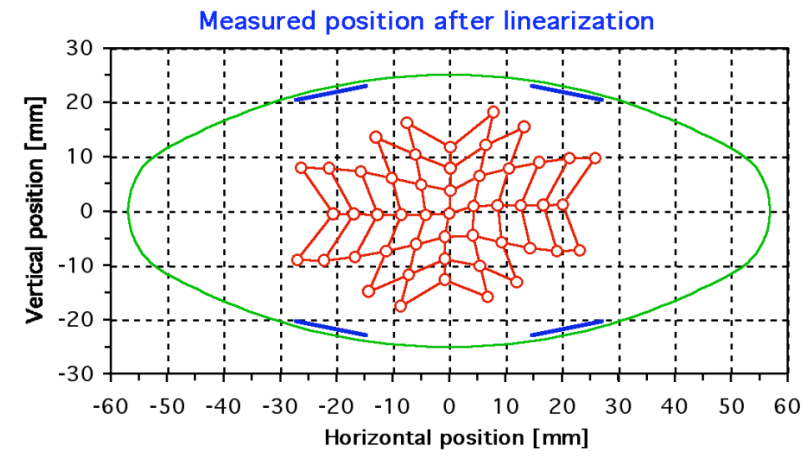

Figure 7. Result of bench-top grid-point measurement of Main Injector BPM response, against BPM hardware cross-section. The non-linear aspect of the measurement is clearly visible.

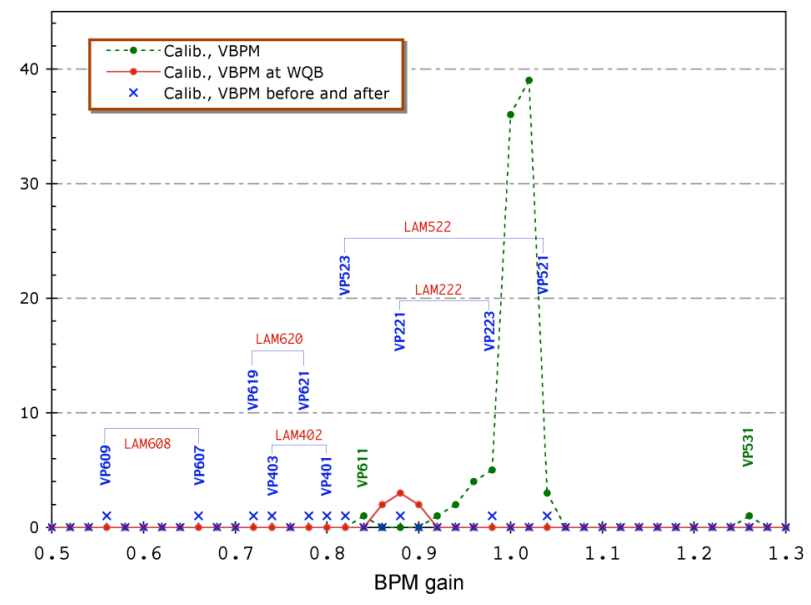

Figure 8. Distribution of Main Injector vertical plane BPM gain calibration plotted in three categories.

The result of Main Injector vertical BPM gain analysis is shown in Figure 8, in three categories. The first category is plotted in green and includes the majority of BPMs. Its distribution peaks at 1.02 with a width of $4 \%$ FWHM, or $\sim 1.7 \%$ in RMS. The second category is plot- ted in red and includes all seven of large aperture BPMs, located next to type B wide aperture quadrupoles (WQB). Its peak at 0.9 is substantially different from that of first category and the cause of it was not yet understood.

The third category, plotted in blue, includes only BPMs from locations that are either one half cell before or one half cell after Lambertson magnets. This category is unique for the following reason. At the Lambertson magnet location closed orbit is offset by as much as $25 \mathrm{~mm}$ such that the circulating beam goes through field free region. Consequently at half cell from Lambertson magnet, where vertical BPMs are located, orbit is offset horizontally. The offsets vary from location to location and are listed in column two of Table I. Column three lists the results of gain analysis, as plotted in Figure 8. Column four lists the expected gain calibration error, based on bench-top measurement and the fact that BPM system assume zero offset in the horizontal plane. While correlations are found in both column three and four, the differences in the correction factors still not understood.

Table I Vertical BPM gain vs. horizontal displacements.

\begin{tabular}{|c|c|c|c|}
\hline $\begin{array}{c}\text { Vertical } \\
\text { BPM }\end{array}$ & $\Delta \mathrm{x}, \mathrm{mm}$ & $\begin{array}{l}\text { Gain, } \\
\text { measured }\end{array}$ & $\begin{array}{l}\text { Gain, } \\
\text { expected }\end{array}$ \\
\hline VP221 & -5.59 & 0.866 & 0.955 \\
\hline VP223 & -4.83 & 0.976 & 0.961 \\
\hline VP401 & 11.27 & 0.797 & 0.837 \\
\hline VP403 & 11.03 & 0.731 & 0.842 \\
\hline VP521 & 11.32 & 1.021 & 0.836 \\
\hline VP523 & 9.88 & 0.819 & 0.866 \\
\hline VP607 & 13.45 & 0.641 & 0.792 \\
\hline VP609 & 16.65 & 0.558 & 0.741 \\
\hline VP619 & 12.05 & 0.718 & 0.821 \\
\hline VP621 & 10.44 & 0.771 & 0.855 \\
\hline
\end{tabular}

\section{CONCLUSION}

The result demonstrated that the relative gain variation of the Fermilab Recycler ring BPM system is at a level of $2 \%$ RMS. It has correctly identified large aperture BPMs from that of the regulars, every time. It also identified BPMs in Main Injector locations where beam position is substantially offset in the orthogonal plane. Their nonlinear nature has been expected but was, understandably, ignored. The significance of this success is not limited to the calibration procedure itself. It is also a validation of TBT lattice function analysis, which provided beta function at BPM locations, at comparable level of accuracy.

The importance of a BPM system in providing excellent position resolution can not be emphasized enough. The same procedure applied to both Recycler ring and Main Injector ring prior to their system upgrade produced results that did not provide much insight.

\section{REFERENCES}

[1] M.J. Yang, "Lattice Measurement with TBT BPM Data", PAC 1995, pp. 2500.

[2] J. Fitzgerald, et al, AIP Conf. Proc., Dec. 10, 1998, Vol. 451 , pp. 370. 